\title{
Impact of COVID-19 pandemic on psychological health and quality of sleep: An online survey from Kolkata
}

\author{
Arijit Mondal', Indranil Saha², Niladri Banerjee ${ }^{3}$, Pradeep Kumar Saha ${ }^{4}$, Soumi Ghosh ${ }^{5}$, \\ Dwaipayan Saha ${ }^{6}$ \\ ${ }^{1}$ Senior Resident, ${ }^{4}$ Professor and Director, ${ }^{5}$ Junior Resident, ${ }^{6}$ Medical Officer, Institute of Psychiatry - A Centre of \\ Excellence, 7 D L Khan Road, Kolkata 700 025, West Bengal, India, ${ }^{2}$ Associate Professor, Department of Psychiatry, \\ Medical College, Kolkata, West Bengal, India, ${ }^{3}$ Senior Resident, Department of Community Medicine, Burdwan Medical \\ College, Burdwan, West Bengal, India
}

Background: As a preventive measure to combat COVID-19 pandemic, India has undergone "lockdown" since March 25, 2020. The pandemic and lockdown can impose a psychological impact in the form of fear, anxiety, stress and insomnia etc. Aims and Objective: The aim of the current study is to assess the magnitude of anxiety, depression, stress and poor sleep quality among the adult population of Kolkata, West Bengal amidst COVID-19 pandemic. Materials and Methods: By this online survey using Google forms, a total of 435 responses were received. The online questionnaire comprises of Socio-demographic characteristics, COVID-19 Anxiety Scale (CAS), Perceived Stress Scale 4 (PSS 4), Patient Health Questionnaire-9 (PHO 9) and Insomnia Severity Index (ISI). Results: COVID-19 related anxiety were almost similar across age groups, sex, marital and educational status. PSS score was found higher in $\leq 25$ years and $>40$ years age group, those not currently married, students and homemakers. Patient Health Questionnaire scores were found significantly different across all background characteristics categories except for the level of education whereas Insomnia Severity index Scale scores were found significantly more among subjects not currently married and among subjects who were working from home. Conclusions: The current study provides evidence towards the negative psychological impact of the pandemic and reminds us about the need for widespread psychiatric services during and after pandemic.

Key words: COVID 19; Pandemic; Psychological health; Sleep Quality; West Bengal

\section{Access this article online}

\section{Website:}

http://nepjol.info/index.php/AJMS DOI: 10.3126/ajms.v12i10.38024 E-ISSN: 2091-0576 P-ISSN: 2467-9100

Copyright (c) 2021 Asian Journal of Medical Sciences

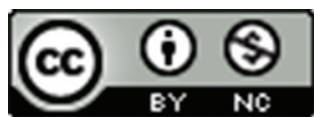

This work is licensed under a Creative Commons Attribution-NonCommercial 4.0 International License.

\section{INTRODUCTION}

On $30^{\text {th }}$ January 2020, the Director-General of World Health Organization (WHO) declared the novel coronavirus (COVID-19) outbreak a public health emergency of international concern (PHEIC), WHO's highest level of alarm. ${ }^{1}$ Till $4^{\text {th }}$ June 2021 , more than two hundred countries have been affected by COVID-19 pandemic with more than 171 million confirmed cases and 3.6 million death. ${ }^{2}$ At the same time India is dealing with the burden of more than 28 million confirmed cases and death toll of nearly three and half lakhs. West Bengal, the site of our current study, has confirmed 14 lacs COVID-19 cases with more than fifteen thousand deaths. ${ }^{3}$ Uncertainty of the trajectory of COVID - 19 pandemic and unpredictable nature of this situation leads to psychological distress - depression, anxiety and traumatic stress. ${ }^{4}$

To combat the rapid rise of cases and to prevent community transmission, Government of India declared nationwide lockdown from midnight of March 25, 2020 with the assurance that the basic needs of general population will be taken care of. Currently, India is undergoing stepwise unlock to normalize the situation apart from the containment zone since $1^{\text {st }}$ June $2020 .{ }^{5}$ Lockdown is a strategy for maintain social distancing to tackle the 
rapid spread of COVID - 19 virus. At the same time, confinement in home for a prolonged time with few close contacts, deviation from normal daily routine, dearth of outdoor leisure activities, excessive use of smart phone and television, information regarding COVID - 19 related morbidity and mortality in media - all these factors lead to greater psychological impact on general population.

In previous studies, psychological impact of isolation and quarantine were assessed during outbreak of Severe Acute Respiratory Syndrome (SARS), influenza, Ebola etc. and short term and long term health problems were seen as consequences. Social isolation was found to be associated with significant health problems ranging from anxiety, fear, depressive symptoms, disturbed sleep in the immediate few days of isolation to symptoms of post traumatic stress disorder and depressive disorder afterwards. ${ }^{6}$ Another study compared quarantined versus non-quarantined individuals during an equine influenza outbreak, reporting a higher level of anxiety and depression (34\%) during the outbreak compared with only $12 \%$ in non-quarantined individuals. ${ }^{7}$

A recent study from India reported prevalence of anxiety, depression and stress as $38.2 \%, 10.5 \%$ and $74.1 \%$ respectively among the general population during lockdown period. ${ }^{8}$ Another online survey from India concluded that almost one third respondent had significant psychological impact. ' Anxiety level was identified to be very high in an online survey due to preoccupation with getting COVID-19 infection. ${ }^{10}$ Survey on general population of a neighboring country reports prevalence of anxiety symptoms, depressive symptoms and stress were $33.7 \%$, $57.9 \%$ and 59.7 respectively. ${ }^{11}$

Generally, stress has an inverse relationship with sleep, but not always. The consequence of stress on sleep duration, timing and quality of sleep is determined by sleep reactivity. ${ }^{12}$ Subjects with high sleep reactivity develop insomnia whereas others do not, even in stressful situations. So, altered daily routine during home confinement due to lockdown and psychological stress during COVID - 19 pandemic enhance the chance of insomnia and disturbed sleep biologically. A nationwide survey from India reported deterioration in sleep quality in the form of shift to later bedtime and waking time, reduction of night-time sleep and increase in daytime napping. Reduction in sleep duration was also found to be associated with depressive symptoms. ${ }^{13}$

In the previous studies, it is obvious that COVID-19 pandemic has immense psychological impact in the form of anxiety, depression and stress and significant alteration of quality and quantity of sleep. All the previous studies were conducted during nationwide lockdown period. In the current scenario [unlock phase], people are likely to have greater stress and psychological morbidity as they are trying to revert back to their earlier lifestyle while the threat of COVID-19 is still ongoing. With this background, the study aims to estimate the magnitude of anxiety, depression and stress among adult residents of Kolkata, West Bengal amidst COVID-19 pandemic, to study the quality of sleep among them and to find out interrelationship between anxiety, depression, stress and sleep quality.

\section{MATERIALS AND METHODS}

Study type and design: It was an observational descriptive study with cross-sectional design.

Study area and study population: The target population was adult residents of Kolkata, West Bengal. Eligible subjects were who can read and comprehend English and who consented to take part in the study.

Study duration: The data collection for the study was done during September 2020-October 2020.

Sample size and sampling: Considering the prevalence of anxiety to be $38.5 \%$ during COVID lockdown according to a study by Grover et. al. ${ }^{8}$, taking $5 \%$ level of significance, and absolute precision of $5 \%$, and $10 \%$ non response, the minimum sample size comes to 421. A sampling frame was prepared with electoral roll and availability of valid mobile numbers. 500 individuals were selected by simple random sampling from that sampling frame through computer generated random numbers. From the selected 500 subjects, 10 blocks of fifty individuals were created. Individuals on each block were communicated over telephone on consecutive days and consent was sought to take part in the study after explaining its nature and methods. Consenting individuals were asked for their e-mail id or WhatsApp number and an online questionnaire was sent to their respective addresses or number through a web-link. They were also briefed about the instructions for completing the questionnaire. The questionnaire was prepared as Google Forms which offers the advantage of limiting to one response and submitting the response maintaining anonymity. A total of 435 complete responses were received.

Study tools:

(i) Sociodemographic Questionnaire: Age, sex, marital status, level of education, occupational status and level of work at the time of data collection were assessed.

(ii) COVID - 19 Anxiety Scale: It is a 7 item questionnaire with good internal consistency reliability [Cronbach's Alpha 0.736]. It is rapidly administrable, valid, and reliable tool that can be used to measure COVID-19 related anxiety among Indian population. ${ }^{14}$ 
(iii) Perceived Stress Scale: To assess the impact of prevailing conditions in emotions, fouritem perceived stress scale4 was used in this study. It has been validated as a measure of perceived stress among various populations and its score moderately correlates with anxiety and depression scale. It has an internal consistency of $0.74 .{ }^{15}$

(iv) Patient Health Questionnaire-9: The Patient Health Questionnaire (PHQ) is a self-administered version of the PRIME-MD diagnostic instrument for common mental disorders. The PHQ-9 is the depression module, which scores each of the 9 Diagnostic and Statistical Manual-IV criteria as "0" (not at all) to "3" (nearly every day). This questionnaire is found to have excellent reliability and validity, and sensitivity and specificity of $88 \%$ for major depression. ${ }^{16}$

(v) Insomnia Severity Index (ISI): Insomnia was assessed using the Insomnia Severity Index (ISI). It has seven items that enquire about nighttime sleep as well as daytime functioning and quality of sleep. Each item is scored on a fivepoint Likert scale with scores ranging between 0 and 28 . Score $>14$ denotes clinical insomnia. ${ }^{17}$

\section{Statistical analysis}

Data were extracted from Google Spread-sheet generated through the questionnaire associated Google form and it was analyzed in SPSS v.20. Data measured in continuous scale were expressed as median and inter quartile range. Normality of data was checked by Shapiro Wilk test and by visualization through histogram and Q-Q plot. Categorical data were expressed as frequency and percentage. Obtained scores were compared across the categories imparted by background characteristics of the participants and differences between categories were tested by Mann Whitney U or Kruskal Wallis test. Screened presence of depression and insomnia were predicted from socio-demographic characteristics through binary logistic regression model after checking for linear relationship between logit of the outcome variables and predictors, absence of multicolinearity and influential points. Monotonicity between age of the study participants and obtained scores of the four scales applied were seen through scatter diagrams and non parametric correlations.

Ethics: Ethics clearance was taken from Institutional Ethics Committee, Institute of Post Graduate Medical Education and Research, Kolkata, vide Memo No. IPGME and R/ IEC/2020/636 dated 09.09.2020.

\section{RESULTS}

The study screens for the magnitude of anxiety related to COVID 19, perceived stress, depression and insomnia in the study population through scales, validated previously in similar context. Obtained scores were compared across the categories imparted by background characteristics of the participants and the screened presence of depression and insomnia were predicted from socio-demographic characteristics. Monotonicity between age of the study participants and obtained scores of the four scales applied were seen through scatter diagrams and non parametric correlations.

Age of the study participants ranged between 18-74 years. $63.2 \%$ respondents were aged between $26-40$ years, $22.8 \%$ were 25 years or less and $14 \%$ were more than 40 years old. $40 \%$ of the participants were female. $49.7 \%$ were currently married. Only $7.4 \%$ of the respondents were undergraduate, whereas majority $(47.8 \%)$ of them had completed their post graduate education. Apart from $8.7 \%$ respondents who were not employed in any specific job and mostly were homemakers, $20.2 \%$ were students, $36.8 \%$ were in involved in private sector, $27.4 \%$ in Govt. sector and $6.9 \%$ were self employed. $21.8 \%$ were not working at the time of data collection; among the rest, $31.5 \%$ were going to work for usual hours and $24.4 \%$ were working from home (Table 1).

Although the COVID anxiety scores were found almost similar across age groups, sex, marital and educational status, the median score was significantly high ( $\mathrm{p}$-value: 0.016) for subjects working from home, especially, for more than usual hours, than the respondents who were going to work; and also high for those who were self-employed or working in private sector than who were students or employed in Govt. sector (p-value: 0.027). Scores of Perceived Stress scale was found significantly different across categories of age, marital status, occupational status and level of work, indicating higher scores in $\leq 25 \mathrm{yrs}$. and $>40$ yrs. age group, among those who were not currently married, among students and homemakers and among those who were not working at that time or working from home or working outside for more than usual hours. Public Health Questionnaire scores were found significantly different across all background characteristics categories except for the level of education whereas Insomnia Severity index Scale found significantly more insomnia score among subjects not currently married (Q1:3, Q2:7, Q3:13) than those who were so (Q1:2, Q2:5, Q3:10) and among subjects working from home (Table 1).

Age in years shown weak negative but significant monotonicity with PSS (rho $=-0.143)$, PHQ (rho $=-0.191)$ and ISI scores (rho $=-0.127)$. Moderate positive correlation of significance were seen between scores of all scales where strongest positive relationship was seen between PHQ and ISI scores $($ rho $=0.623)$ (Figure 1).

Asian Journal of Medical Sciences | Oct 2021 | Vol 12 | Issue 10 


\begin{tabular}{|c|c|c|c|c|c|c|c|c|c|}
\hline \multirow[t]{2}{*}{ Characteristics } & \multirow[t]{2}{*}{$\begin{array}{l}\text { Frequency } \\
\qquad(\%)\end{array}$} & $\begin{array}{c}\text { CAS } \\
\text { score }^{\$}\end{array}$ & \multirow[t]{2}{*}{ p-value } & \multirow{2}{*}{$\begin{array}{c}\text { PSS } \\
\text { score }^{\$ \$} \\
\begin{array}{c}\text { Madian } \\
\text { (IQR) }\end{array}\end{array}$} & \multirow[t]{2}{*}{ p-value $\#$} & \multirow{2}{*}{$\begin{array}{c}\text { PHQ } \\
\text { score }^{\$ \$} \\
\begin{array}{c}\text { Madian } \\
\text { (IQR) }\end{array}\end{array}$} & \multirow[t]{2}{*}{ p-value } & $\begin{array}{c}\text { ISI } \\
\text { score }^{\$ \$ \$ \$}\end{array}$ & \multirow[t]{2}{*}{ p-value } \\
\hline & & $\begin{array}{l}\text { Madian } \\
\text { (IQR) }\end{array}$ & & & & & & $\begin{array}{c}\text { Madian } \\
\text { (IQR) }\end{array}$ & \\
\hline \multicolumn{10}{|l|}{ Age } \\
\hline $26-40$ & $275(63.2)$ & $\begin{array}{c}16 \\
(13-19)\end{array}$ & & $\begin{array}{c}7 \\
(5-8)\end{array}$ & & $\begin{array}{c}4 \\
(2-7)\end{array}$ & & $\begin{array}{c}6 \\
(2-11)\end{array}$ & \\
\hline$>40$ & $61(14.0)$ & $\begin{array}{c}16 \\
(12-20)\end{array}$ & & $\begin{array}{c}8 \\
(5-8)\end{array}$ & & $\begin{array}{c}4 \\
(2-9)\end{array}$ & & $\begin{array}{c}7 \\
(2-11)\end{array}$ & \\
\hline \multicolumn{10}{|l|}{ Sex } \\
\hline Female & $174(40.0)$ & $\begin{array}{c}16 \\
(13-20)\end{array}$ & 0.311 & $\begin{array}{c}7 \\
(5-9)\end{array}$ & 0.349 & $\begin{array}{c}5 \\
(3-10)\end{array}$ & 0.001 & $\begin{array}{c}7 \\
(3-13)\end{array}$ & 0.050 \\
\hline \multicolumn{10}{|l|}{ Marital status } \\
\hline Currently married & $216(49.7)$ & $\begin{array}{c}16 \\
(14-19)\end{array}$ & 0.051 & $\begin{array}{c}7 \\
(5-8)\end{array}$ & 0.002 & $\begin{array}{c}4 \\
(2-8)\end{array}$ & 0.002 & $\begin{array}{c}5 \\
(2-10)\end{array}$ & 0.010 \\
\hline Others & $219(50.3)$ & $\begin{array}{c}16 \\
(12-19)\end{array}$ & & $\begin{array}{c}8 \\
(6-10)\end{array}$ & & $\begin{array}{c}6 \\
(3-10)\end{array}$ & & $\begin{array}{c}7 \\
(3-13)\end{array}$ & \\
\hline \multicolumn{10}{|l|}{ Educational status } \\
\hline Undergraduate & $32(7.4)$ & $\begin{array}{c}17 \\
(14-20)\end{array}$ & 0.910 & $\begin{array}{c}8 \\
(6-11)\end{array}$ & 0.178 & $\begin{array}{c}6 \\
(3-12)\end{array}$ & 0.112 & $\begin{array}{c}7 \\
(2-14)\end{array}$ & 0.712 \\
\hline Graduate & $195(44.8)$ & $\begin{array}{c}16 \\
(12-19)\end{array}$ & & $\begin{array}{c}7 \\
(5-9)\end{array}$ & & $\begin{array}{c}4 \\
(2-8)\end{array}$ & & $\begin{array}{c}6 \\
(2-12)\end{array}$ & \\
\hline Postgraduate and above & $208(47.8)$ & $\begin{array}{c}16 \\
(13-19)\end{array}$ & & $\begin{array}{c}7 \\
(6-9)\end{array}$ & & $\begin{array}{c}5 \\
(2-8)\end{array}$ & & $\begin{array}{c}6 \\
(3-11)\end{array}$ & \\
\hline \multicolumn{10}{|l|}{ Occupational status } \\
\hline Govt. Sector & $119(27.4)$ & $\begin{array}{c}15 \\
(12-19)\end{array}$ & 0.027 & $\begin{array}{c}7 \\
(5-8)\end{array}$ & 0.033 & $\begin{array}{c}4 \\
(2-8)\end{array}$ & 0.004 & $\begin{array}{c}5 \\
(2-10)\end{array}$ & 0.538 \\
\hline Self employed & $30(6.9)$ & $\begin{array}{c}17 \\
(14-19)\end{array}$ & & $\begin{array}{c}7 \\
(5-8)\end{array}$ & & $\begin{array}{c}4 \\
(2-7)\end{array}$ & & $\begin{array}{c}6 \\
(2-10)\end{array}$ & \\
\hline Student & $88(20.2)$ & $\begin{array}{c}15 \\
(12-20)\end{array}$ & & $\begin{array}{c}8 \\
(5-10)\end{array}$ & & $\begin{array}{c}6 \\
(3-11)\end{array}$ & & $\begin{array}{c}7 \\
(2-15)\end{array}$ & \\
\hline At home & $38(8.7)$ & $\begin{array}{c}16 \\
(14-19)\end{array}$ & & $\begin{array}{c}8 \\
(6-9)\end{array}$ & & $\begin{array}{c}5 \\
(3-8)\end{array}$ & & $\begin{array}{c}7 \\
(5-12)\end{array}$ & \\
\hline \multicolumn{10}{|l|}{ Level of work } \\
\hline Not working at present & $95(21.8)$ & $\begin{array}{c}16 \\
(13-19)\end{array}$ & 0.016 & $\begin{array}{c}8 \\
(6-10)\end{array}$ & 0.001 & $\begin{array}{c}6 \\
(3-10)\end{array}$ & 0.000 & $\begin{array}{c}7 \\
(2-12)\end{array}$ & 0.000 \\
\hline $\begin{array}{l}\text { Working from home for } \\
\text { less than usual hours }\end{array}$ & $37(8.5)$ & $\begin{array}{c}17 \\
(15-20)\end{array}$ & & $\begin{array}{c}8 \\
(6-10)\end{array}$ & & $\begin{array}{c}7 \\
(5-14)\end{array}$ & & $\begin{array}{c}10 \\
(2-14)\end{array}$ & \\
\hline $\begin{array}{l}\text { Working from home for } \\
\text { usual hours }\end{array}$ & $32(7.4)$ & $\begin{array}{c}17 \\
(13-19)\end{array}$ & & $\begin{array}{c}8 \\
(6-9)\end{array}$ & & $\begin{array}{c}6 \\
(4-9)\end{array}$ & & $\begin{array}{c}8 \\
(4-13)\end{array}$ & \\
\hline $\begin{array}{l}\text { Working from home for } \\
\text { more than usual hours }\end{array}$ & $37(8.5)$ & $\begin{array}{c}18 \\
(16-21)\end{array}$ & & $\begin{array}{c}8 \\
(6-10)\end{array}$ & & $\begin{array}{c}7 \\
(3-10)\end{array}$ & & $\begin{array}{c}9 \\
(5-14)\end{array}$ & \\
\hline $\begin{array}{l}\text { Going to work for less } \\
\text { than usual hours }\end{array}$ & $51(11.7)$ & $\begin{array}{c}17 \\
(12-19)\end{array}$ & & $\begin{array}{c}7 \\
(4-8)\end{array}$ & & $\begin{array}{c}4 \\
(1-7)\end{array}$ & & $\begin{array}{c}6 \\
(2-11)\end{array}$ & \\
\hline $\begin{array}{l}\text { Going to work for usual } \\
\text { hours }\end{array}$ & $137(31.5)$ & $\begin{array}{c}16 \\
(13-19)\end{array}$ & & $\begin{array}{c}7 \\
(5-8)\end{array}$ & & $\begin{array}{c}3 \\
(2-6)\end{array}$ & & $\begin{array}{c}4 \\
(2-8)\end{array}$ & \\
\hline $\begin{array}{l}\text { Going to work for more } \\
\text { than usual hours }\end{array}$ & $46(10.6)$ & $\begin{array}{c}15 \\
(12-18)\end{array}$ & & $\begin{array}{c}8 \\
(6-9)\end{array}$ & & $\begin{array}{c}5 \\
(2-7)\end{array}$ & & $\begin{array}{c}6 \\
(3-10)\end{array}$ & \\
\hline
\end{tabular}

s: COVID Anxiety Scale, maximum score: 28 , minimum: 7

ss: Perceived Stress Scale (4 item), maximum score: 16 , minimum: 0

sss: Patient Health Questionnaire (PHQ-9); maximum score: 27 , minimum: 0

ssss: Insomnia Severity Index; maximum score: 28 , minimum: 0

\#: Mann-Whitney U test was applied for variables: Sex and Marital Status. For all other variables, Kruskal-Wallis test was used

Screen positivity for presence of depression and insomnia were considered as per scalar instructions. More depression was noted among- subjects aged 25 yrs. or less (35.4\%), females (26.4\%), currently un-marrieds $(25.1 \%)$, undergraduates $(31.3 \%)$, students (30.7\%) and among those who were working from home for less than usual hours $(43.2 \%)$; however when they were all adjusted for predicting presence of depression in a regression model, only two 


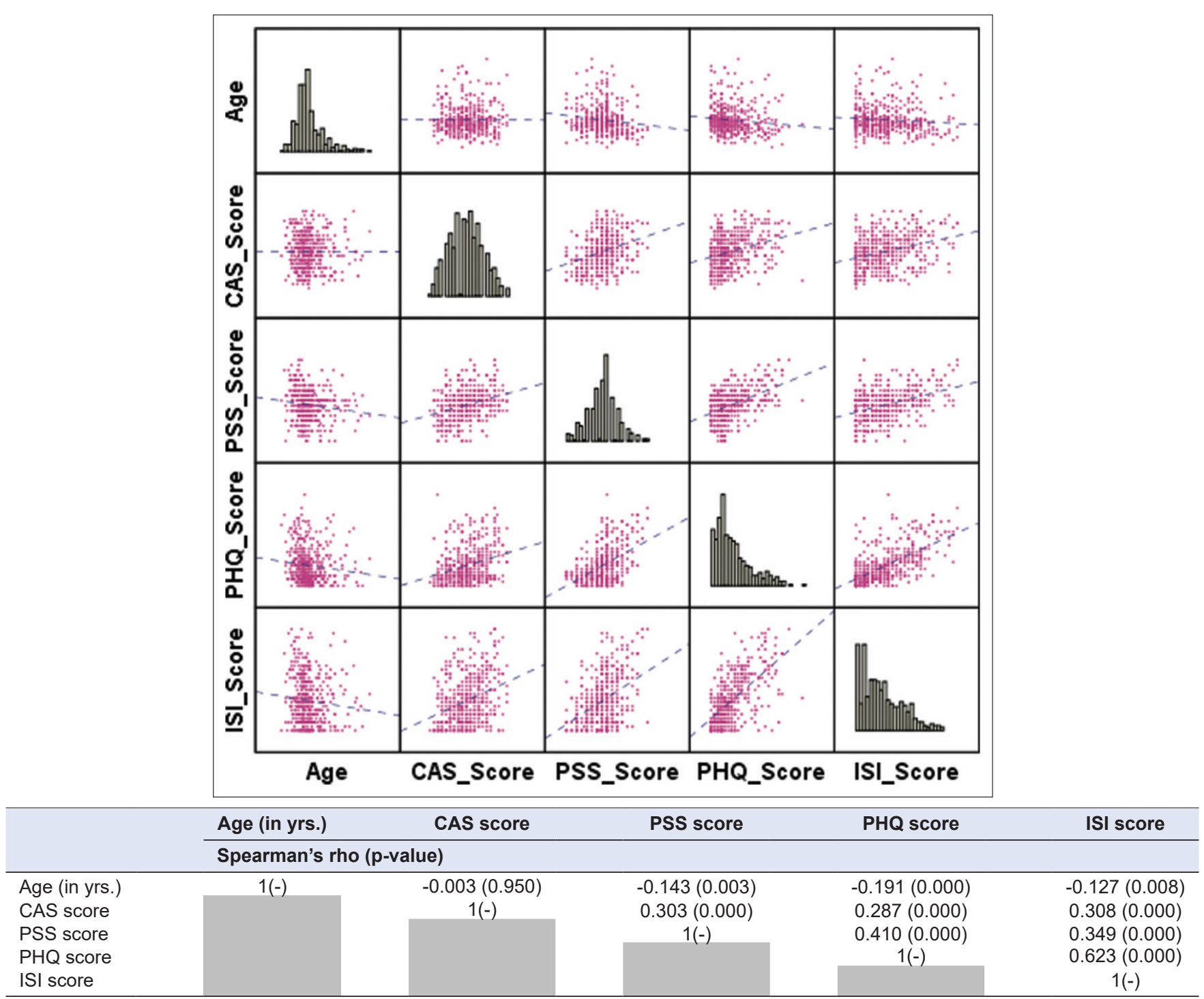

Figure 1: Scatter matrix showing interrelationship between scores obtained for various scales applied, by study population and their age in years $(n=435)$

factors, i.e. age between $26-40$ yrs. $[\mathrm{AOR}=0.426(95 \%$ CI: 0.200-0.905)] and working from home for less than usual hours [2.437 (95\% CI: 1.031-5.761)] were found to be significant predictors of depression. Students were found to be 3.7 (95\% CI: 1.003-13.370) times more likely to have clinical insomnia than subjects who were 'at home'. Albeit being a significant predictor, clinical insomnia was found to be more common among subjects aged $\leq 25 \mathrm{yrs}$. $(24.2 \%)$, females $(17.8 \%)$, currently un-marrieds $(18.3 \%)$, under graduates $(15.6 \%)$, subjects working from home $(24.3 \%$, $18.8 \%, 24.3 \%)$ and students $(26.1 \%$ ) (Table 2).

\section{DISCUSSIONS}

This study was conducted to assess the psychological health of general population of West Bengal during first wave of COVID-19 pandemic. Prevalence of COVID related anxiety symptoms were persistent throughout the whole population irrespective of age, sex and marital status. It is an established fact that mental health related problems like stress and depression are common in female than male ${ }^{18}$ but COVID related anxiety were similar in both sexes in our study. This result goes with study conducted from Andhra Pradesh using CAS scale. ${ }^{19}$ Subjects with undergraduate or below educational status show higher score (though not statistically significant) than graduates and postgraduates. This finding goes with the study finding of Chandu et al., that subjects with higher educational qualification have lesser CAS score. ${ }^{14}$ The median COVID-19 Anxiety Scale score was significantly high for those who were self employed, working in private sector and those who were working from home for more than 


\begin{tabular}{|c|c|c|c|c|c|c|c|}
\hline \multirow[t]{2}{*}{ Characteristics } & \multirow[t]{2}{*}{$\begin{array}{l}\text { Frequency } \\
\quad(\%)\end{array}$} & $\begin{array}{l}\text { Presence of } \\
\text { depression }\end{array}$ & \multirow[t]{2}{*}{$\begin{array}{c}\text { AOR } \\
(95 \% \mathrm{Cl})^{\mathrm{s}}\end{array}$} & \multirow[t]{2}{*}{ p-value ${ }^{s}$} & $\begin{array}{l}\text { Presence of } \\
\text { Insomnia }\end{array}$ & \multirow[t]{2}{*}{$\begin{array}{c}\text { AOR } \\
(95 \% \mathrm{Cl})^{\#}\end{array}$} & \multirow[t]{2}{*}{ p-value ${ }^{\#}$} \\
\hline & & Frequency (\%) & & & \multirow{2}{*}{\multicolumn{3}{|c|}{ Frequency (\%) }} \\
\hline \multicolumn{5}{|l|}{ Age groups (in years) } & & & \\
\hline$\leq 25$ & $99(22.8)$ & $35(35.4)$ & Ref. & - & $24(24.2)$ & Ref. & - \\
\hline $26-40$ & $275(63.2)$ & $43(15.6)$ & $\begin{array}{c}0.426 \\
(0.200-0.905)\end{array}$ & 0.026 & 31 (11.3) & $\begin{array}{c}0.695 \\
(0.301-1.603)\end{array}$ & 0.394 \\
\hline$>40$ & $61(14.0)$ & $12(19.7)$ & $\begin{array}{c}0.644 \\
(0.219-1.891)\end{array}$ & 0.423 & $7(11.5)$ & $\begin{array}{c}0.881 \\
(0.244-3.179)\end{array}$ & 0.846 \\
\hline \multicolumn{8}{|l|}{ Sex } \\
\hline Female & $174(40.0)$ & $46(26.4)$ & $\begin{array}{c}1.423 \\
(0.834-2.427)\end{array}$ & 0.195 & $31(17.8)$ & $\begin{array}{c}1.160 \\
(0.631-2.133)\end{array}$ & 0.633 \\
\hline Male & $261(60.0)$ & $44(16.9)$ & Ref. & - & $31(11.9)$ & Ref. & - \\
\hline \multicolumn{8}{|l|}{ Marital status } \\
\hline Currently married & $216(49.7)$ & $35(16.2)$ & Ref. & - & $22(10.2)$ & Ref. & - \\
\hline Others & $219(50.3)$ & $55(25.1)$ & $\begin{array}{c}1.091 \\
(0.580-2.052)\end{array}$ & 0.786 & $40(18.3)$ & $\begin{array}{c}1.218 \\
(0.588-2.523)\end{array}$ & 0.595 \\
\hline \multicolumn{8}{|l|}{ Educational status } \\
\hline Undergraduate & $32(7.4)$ & $10(31.3)$ & Ref. & - & $5(15.6)$ & Ref. & - \\
\hline Graduate & $195(44.8)$ & $37(19.0)$ & $\begin{array}{c}1.472 \\
(0.534-4.061)\end{array}$ & 0.455 & $25(12.8)$ & $\begin{array}{c}3.126 \\
(0.926-10.555)\end{array}$ & 0.066 \\
\hline $\begin{array}{l}\text { Postgraduate and } \\
\text { above }\end{array}$ & $208(47.8)$ & $43(20.7)$ & $\begin{array}{c}1.447 \\
(0.542-3.863)\end{array}$ & 0.460 & $32(15.4)$ & $\begin{array}{c}3.049 \\
(0.938-9.911)\end{array}$ & 0.064 \\
\hline \multicolumn{8}{|l|}{ Occupational status } \\
\hline Govt. Sector & $119(27.4)$ & $18(15.1)$ & $\begin{array}{c}1.551 \\
(0.516-4.659)\end{array}$ & 0.435 & $14(11.8)$ & $\begin{array}{c}1.781 \\
(0.483-6.563)\end{array}$ & 0.386 \\
\hline Pvt. Sector & $160(36.8)$ & $31(19.4)$ & $\begin{array}{c}2.050 \\
(0.689-6.096)\end{array}$ & 0.197 & $9(11.9)$ & $\begin{array}{c}1.448 \\
(0.387-5.416)\end{array}$ & 0.582 \\
\hline Self employed & $30(6.9)$ & $7(23.3)$ & $\begin{array}{c}2.099 \\
(0.573-7.694)\end{array}$ & 0.263 & $2(6.7)$ & $\begin{array}{c}0.735 \\
(0.116-4.667)\end{array}$ & 0.744 \\
\hline Student & $88(20.2)$ & $27(30.7)$ & $\begin{array}{c}1.752 \\
(0.578-5.308)\end{array}$ & 0.321 & $23(26.1)$ & $\begin{array}{c}3.662 \\
(1.003-13.370)\end{array}$ & 0.049 \\
\hline At home & $38(8.7)$ & $7(18.4)$ & Ref. & - & $4(10.5)$ & Ref. & - \\
\hline \multicolumn{8}{|l|}{ Level of work } \\
\hline Not working at present & $95(21.8)$ & $25(26.3)$ & Ref. & - & $17(17.9)$ & Ref. & - \\
\hline $\begin{array}{l}\text { Working from home for } \\
\text { less than usual hours }\end{array}$ & $37(8.5)$ & $16(43.2)$ & $\begin{array}{c}2.437 \\
(1.031-5.761)\end{array}$ & 0.042 & $9(24.3)$ & $\begin{array}{c}2.123 \\
(0.782-5.761)\end{array}$ & 0.139 \\
\hline $\begin{array}{l}\text { Working from home for } \\
\text { usual hours }\end{array}$ & $32(7.4)$ & $7(21.9)$ & $\begin{array}{c}0.715 \\
(0.244-2.088)\end{array}$ & 0.539 & $6(18.8)$ & $\begin{array}{c}1.241 \\
(0.386-3.993)\end{array}$ & 0.717 \\
\hline $\begin{array}{l}\text { Working from home for } \\
\text { more than usual hours }\end{array}$ & $37(8.5)$ & $11(29.7)$ & $\begin{array}{c}1.277 \\
(0.462-3.524)\end{array}$ & 0.637 & $9(24.3)$ & $\begin{array}{c}2.092 \\
(0.680-6.434)\end{array}$ & 0.198 \\
\hline $\begin{array}{l}\text { Going to work for less } \\
\text { than usual hours }\end{array}$ & $51(11.7)$ & $9(17.6)$ & $\begin{array}{c}0.716 \\
(0.270-1.902)\end{array}$ & 0.503 & $5(9.8)$ & $\begin{array}{c}0.646 \\
(0.196-2.125)\end{array}$ & 0.474 \\
\hline $\begin{array}{l}\text { Going to work for } \\
\text { usual hours }\end{array}$ & $137(31.5)$ & $16(11.7)$ & $\begin{array}{c}0.495 \\
(0.208-1.176)\end{array}$ & 0.111 & $11(8.0)$ & $\begin{array}{c}0.553 \\
(0.206-1.486)\end{array}$ & 0.240 \\
\hline $\begin{array}{l}\text { Going to work for more } \\
\text { than usual hours }\end{array}$ & $46(10.6)$ & $6(13.0)$ & $\begin{array}{c}0.543 \\
(0.183-1.613)\end{array}$ & 0.272 & $5(10.9)$ & $\begin{array}{c}0.737 \\
(0.222-2.439)\end{array}$ & 0.617 \\
\hline
\end{tabular}

s: Multivariable logistic regression, Enter method, Omnibus test p value for model: 0.003, Hosmer Lemeshow test p-value: 0.158, Negelkerke R2: 0.123

\#: Multivariable logistic regression, Enter method, Omnibus test p value for model: 0.027, Hosmer Lemeshow test p-value: 0.269 , Negelkerke R2: 0.114

usual hours. This can be explained by economic security or social security. Probable fatal outcome following infection with COVID-19 is the underlying thought for high level of CAS score among the above mentioned population. Safety, risk of infection, social exclusion, stigma and job insecurity were the main factors for job related stress during COVID-19 pandemic. $^{20}$

Perceived stress scale score was significantly higher in younger age group ( $<25$ years) and elderly ( $>40$ years), single, students and unemployed (staying at home). Severity of stress was less in 25 to 40 years age group may be due to that fact that COVID-19 has less mortality in this age group or that they are mostly working and less affected by home confinement. Stress in younger and elderly may be due to social confinement and decreased interaction with others during lockdown phase or high risk of mortality due to COVID-19 in case of elderly. The imposed confinement may play a role for increased stress among students, unemployed and those who are single. This finding is similar to study from Bangladesh where marked stress and anxiety were noted in young ( $<23$ years) and elderly ( $>50$ years) population, students, subjects with low education and low family income. ${ }^{11}$ Level of stress 
was comparatively low among those going outside for job than among those who were not working or working from home. This may be due to prolonged home confinement and excessive workload, fear of infection at workplace in case of health care worker, police etc.

One fifth $(20.7 \%)$ of the study population has depressive symptoms, which is close to the result $(24.7 \%)$ of index study of psychological impact of COVID-19 from West Bengal. ${ }^{21}$ Cross-sectional web-based survey from China also reports $20.1 \%$ prevalence of depressive symptoms in general population..$^{22}$ Online survey from India, using the same questionnaire, also reports $29 \%$ prevalence of depression (mild to severe) in general population. This study was conducted at the first phase of strict nationwide lockdown when people were unaware about future days and trajectory of COVID-19 pandemic. $^{8}$ The same factor may be the cause of high prevalence of depression (57.9\%) in Bangladesh population. ${ }^{11}$

Frequency of depression was more among subjects below 25 years $(35.4 \%)$, female $(26.4 \%)$, single $(25.1 \%)$, undergraduates $(31.3 \%)$, students $(30.7 \%)$ and those who were working from home for less than usual hours (43.2\%). In Chinese study, younger participants reported significantly higher depressive and anxiety symptoms than older people. ${ }^{22}$ More prevalence of depressive symptoms in female goes with study by Lim et al., ${ }^{18}$ Subjects with undergraduate or below education level, students and subjects working from home less than usual hours develop depressive symptoms, probably due to lack of knowledge about pandemic and its uncertain trajectory, home confinement, deviation from normal daily life, decreased interaction with peer groups and job insecurity.

In our study, $14.25 \%$ of participants have insomnia which is close to the finding $(10 \%)$ of Indian study by Gupta et al., ${ }^{13}$ Study from China also reports prevalence of insomnia $15.3 \%$ using the same scale. ${ }^{23}$ Among all the occupation, students have significant sleep related problem in our study. This may be explained by home confinement, excessive use of smartphone and internet and change in daily routine during lockdown. ${ }^{24}$ Positive relationship between PHQ and ISI scores suggests depressive symptoms and insomnia go hand in hand, as insomnia is a mere presentation of underlying depression. ${ }^{25}$

To conclude, this study provides evidence regarding affection of psychological health of general population and estimates its magnitude in various subgroups. These will help to plan mental health promotive measures for high risk population in face of further waves and lockdowns.

\section{ACKNOWLEDGEMENT}

The authors take this opportunity to thank Institute of Psychiatry - A Centre of Excellence, Kolkata for their whole hearted support for this study.

\section{REFERENCES}

1. Timeline: WHO's COVID-19 response [Internet]. [cited 2021 Jun 4]. Available from: https://www.who.int/emergencies/ diseases/novel-coronavirus-2019/interactive-timeline

2. Coronavirus disease (COVID-19) - World Health Organization [Internet]. [cited 2021 Jun 4]. Available from: https://www.who. int/emergencies/diseases/novel-coronavirus-2019

3. Aarogya Setu [Internet]. [cited 2021 Jun 4]. Available from: https://aarogyasetu.gov.in

4. Zandifar A and Badrfam R. Iranian mental health during the COVID-19 epidemic. Asian J Psychiatry. 2020; 51:101990. https://doi.org/10.1016/j.ajp.2020.101990

5. COVID-19 pandemic lockdown in India. In: Wikipedia [Internet]. 2020 [cited 2020 Dec 24]. Available from: https://en.wikipedia. org/w/index.php?title=COVID-19_pandemic_lockdown_in_India and oldid $=996057503$

6. Reynolds DL, Garay JR, Deamond SL, Moran MK, Gold W and Styra R. Understanding, compliance and psychological impact of the SARS quarantine experience. Epidemiol Infect. 2008; 136(7):997-1007. https://doi.org/10.1017/S0950268807009156

7. Taylor MR, Agho KE, Stevens GJ and Raphael B. Factors influencing psychological distress during a disease epidemic: Data from Australia's first outbreak of equine influenza. BMC Public Health. 2008;8(1):347.

https://doi.org/10.1186/1471-2458-8-347

8. Grover S, Sahoo S, Mehra A, Avasthi A, Tripathi A, Subramanyan A, et al. Psychological impact of COVID-19 lockdown: An online survey from India. Indian J Psychiatry. 2020; 62(4):354.

https://doi.org/10.4103/psychiatry.IndianJPsychiatry_427_20

9. Varshney M, Parel JT, Raizada N and Sarin SK. Initial psychological impact of COVID-19 and its correlates in Indian Community: An online (FEEL-COVID) survey. PLOS ONE. 2020; 29; 15(5):e0233874.

https://doi.org/10.1371/journal.pone.0233874

10. Roy D, Tripathy S, Kar SK, Sharma N, Verma SK \& Kaushal V. Study of knowledge, attitude, anxiety and perceived mental healthcare need in Indian population during COVID-19 pandemic. Asian J Psychiatry. 2020; 102083.

https://doi.org/10.1016/j.ajp.2020.102083

11. Banna MdHA, Sayeed A, Kundu S, Christopher E, Hasan MT, Begum MR, et al. The impact of the COVID-19 pandemic on the mental health of the adult population in Bangladesh: a nationwide cross-sectional study. Int J Environ Health Res. 2020; 1-12. https://doi.org/10.31234/osf.io/chw5d

12. Kalmbach DA, Cuamatzi-Castelan AS, Tonnu CV, Tran KM, Anderson JR, Roth T, et al. Hyperarousal and sleep reactivity in insomnia: current insights. Nat Sci Sleep. 2018;10:193-201. https://doi.org/10.2147/NSS.S138823

13. Gupta R, Grover S, Basu A, Krisnan V, Tripathi A, Subramanyam A, et al. Changes in sleep pattern and sleep quality during COVID-19 lockdown. Indian J Psychiatry. 2020; 62(4):370. 
https://doi.org/10.4103/psychiatry.IndianJPsychiatry_523 20

14. Chandu VC, Pachava S, Vadapalli V and Marella Y. Development and Initial Validation of the COVID-19 Anxiety Scale. Indian J Public Health. 2020; 64(6):201.

https://doi.org/10.4103/ijph.IJPH_492_20

15. Frontiers | Determining Factors for Stress Perception Assessed with the Perceived Stress Scale (PSS-4) in Spanish and Other European Samples | Psychology [Internet]. [cited 2020 Nov 27]. Available from: https://www.frontiersin.org/articles/10.3389/ fpsyg.2018.00037/full

16. Kroenke K, Spitzer RL and Williams JB. The PHQ-9: validity of a brief depression severity measure. J Gen Intern Med. 2001; 16(9):606-613.

https://doi.org/10.1046/j.1525-1497.2001.016009606.x

17. Gagnon $\mathrm{C}$, Bélanger L, Ivers $\mathrm{H}$ and Morin $\mathrm{CM}$. Validation of the Insomnia Severity Index in primary care. J Am Board Fam Med JABFM. 2013; 26(6):701-710.

https://doi.org/10.3122/jabfm.2013.06.130064

18. Lim GY, Tam WW, Lu Y, Ho CS, Zhang MW and Ho RC Prevalence of Depression in the Community from 30 Countries between 1994 and 2014. Sci Rep. 2018; 8(1):2861.

https://doi.org/10.1038/s41598-018-21243-x

19. Meesala N, Harsha GD, Kandikatla P, Karteekvarma Pv, Nadakuditi $\mathrm{S}$ and Kakaraparthi $\mathrm{S}$. Measuring the impact of COVID-19 on mental health as a preliminary procedure in primary care provision: A cross-sectional study using COVID-19 anxiety scale. J Fam Med Prim Care. 2020; 9(11):5554. https://doi.org/10.4103/jfmpc.jfmpc_1244_20
20. Hamouche S. COVID-19 and employees' mental health: stressors, moderators and agenda for organizational actions. Emerald Open Res. 2020; 2:15. https://doi.org/10.35241/emeraldopenres.13550.1

21. Chakraborty $\mathrm{K}$ and Chatterjee $\mathrm{M}$. Psychological impact of COVID-19 pandemic on general population in West Bengal: A cross-sectional study. Indian J Psychiatry. 2020; 62(3):266-272. https://doi.org/10.4103/psychiatry.IndianJPsychiatry_276_20

22. Huang $Y$ and Zhao N. Generalized anxiety disorder, depressive symptoms and sleep quality during COVID-19 outbreak in China: a web-based cross-sectional survey. Psychiatry Res. 2020;288:112954. https://doi.org/10.1016/j.psychres.2020.112954

23. Tan W, Hao F, McIntyre RS, Jiang L, Jiang X, Zhang L, et al. Is returning to work during the COVID-19 pandemic stressful? A study on immediate mental health status and psychoneuroimmunity prevention measures of Chinese workforce. Brain Behav Immun. 2020; 87:84-92. https://doi.org/10.1016/j.bbi.2020.04.055

24. Alshobaili $F$ and AlYousefi $N$. The effect of smartphone usage at bedtime on sleep quality among Saudi non- medical staff at King Saud University Medical City. J Fam Med Prim Care. 2019; 8(6):1953. https://doi.org/10.4103/jfmpc.jfmpc_269_19

25. Isaac F and Greenwood K. The relationship between insomnia and depressive symptoms: genuine or artifact? Neuropsychiatr Dis Treat. 2011; 57. https://doi.org/10.2147/NDT.S16267

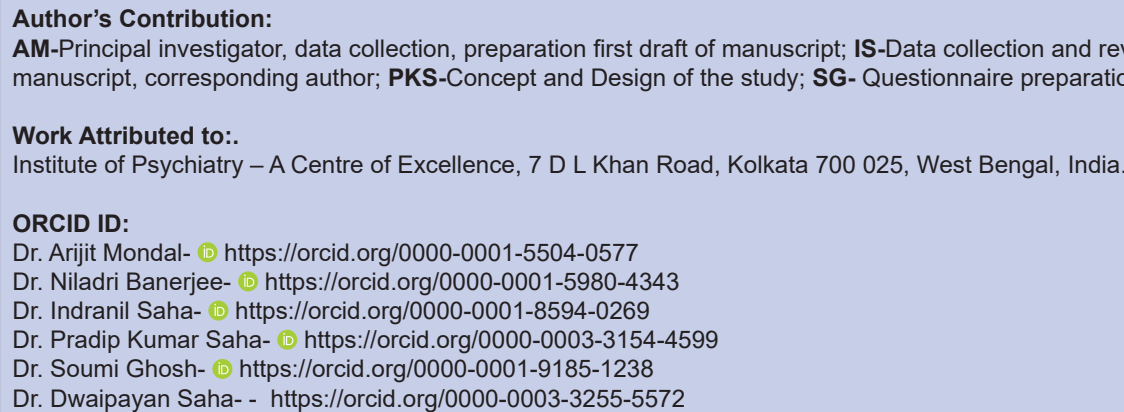

